\title{
Tumor cell-specific chromosomal abnormality in the vascular endothelial cells of anaplastic oligodendroglioma
}

\author{
Haihui Jiang, MD, ${ }^{1}$ Zhe Zhang, MD, ${ }^{1}$ Xiaohui Ren, MD, ${ }^{1}$ Wei Zeng, MD, ${ }^{1}$ Junmei Wang, MD, ${ }^{2}$ and \\ Song Lin, MD' \\ 1Department of Neurosurgery, Beijing Tiantan Hospital, Capital Medical University, China National Clinical Research Center for \\ Neurological Diseases, Center of Brain Tumor, Beijing Institute for Brain Disorders and Beijing Key Laboratory of Brian Tumor; \\ and ${ }^{2}$ Department of Pathology, Beijing Neurosurgical Institute, Capital Medical University, Beijing, China
}

\begin{abstract}
OBJECTIVE $1 p / 19 q$ co-deletion is a well-established tumor cell-specific chromosomal abnormality in oligodendroglial tumors. The endothelial cells (ECs) of oligodendroglial tumor vessels are considered to be normal cells that do not acquire mutations.

METHODS A total of 30 samples from 16 male and 14 female patients (median age of 46.5 years) with a histological diagnosis of primary anaplastic oligodendroglioma $(\mathrm{AO})$ were collected in the study. The immunofluorescence technique was used to identify vascular ECs, and the $1 p / 19 q$ status was detected with fluorescence in situ hybridization. KaplanMeier plots were compared using the log-rank method.

RESULTS The ECs in AO had a higher $1 p 36$ (detected signal) deletion rate than $1 q 25$ (reference signal) $(p<0.01)$ and a higher 19q13 (detected signal) deletion rate than 19p13 (reference signal) $(p<0.01)$. The survival analysis results showed that both the progression-free survival (PFS) and overall survival (OS) of the patients with 1p/19q-co-deleted ECs were significantly longer than those with 1p/19q-intact ECs (PFS, $p<0.001 ; 0 S, p<0.001$ ). This correlation was validated by an independent cohort. In addition, the Cox regression model revealed that $1 \mathrm{p} / 19 \mathrm{q}$ co-deletion in ECs was an independent prognostic factor (HR 0.056 [95\% Cl 0.012-0.261], p < 0.001 for PFS; HR 0.061 [95\% Cl 0.013-0.280], $p<0.01$ for OS).
\end{abstract}

CONCLUSIONS 1p/19q co-deletion and polysomy can be also found in the ECs of $A O$, which suggests that the ECs are, in part, tumor related and reflect a novel aspect of tumor angiogenesis.

http://thejns.org/doi/abs/10.3171/2015.8.JNS15879

KEY WORDS anaplastic oligodendroglioma; 1p/19q; endothelial cells; angiogenesis; prognosis; oncology

$\mathrm{G}$ LIOMAS are the most common type of malignant primary intracranial tumors and account for almost $80 \%$ of primary malignant brain tumors..$^{14}$ Despite multimodal aggressive treatment, which consists of resection, local radiotherapy, and systemic chemotherapy, the prognosis of gliomas is still unsatisfying. ${ }^{8}$ Targeting endothelial cells (ECs) was a major focus of antiangiogenic therapeutics, which was considered the most promising treatment in past years. However, most current therapies that target glioma ECs have been proven to be effectlimited. On the contrary, some experts hold that, to some extent, they may transform tumor growth patterns toward a more invasive phenotype. ${ }^{11}$ The most widely used antiangiogenic drug for gliomas - bevacizumab, a human- ized monoclonal antibody against the vascular endothelial growth factor A ligand - has not reached the expected clinical effect..$^{5,7}$ Therefore, in order to pursue optimal antiangiogenic benefits, further insights into glioma vascular development and maintenance would have profound translational implications.

In the present study, for the first time, we found that the ECs of oligodendroglial tumors also harbored 1p/19q codeletion, which was a recognized molecular biomarker for oligodendroglial tumor cells. These findings implied that ECs and tumor cells in gliomas shared a common pathway in tumor genesis. Here, we propose a hypothesis that ECs might differentiate from the glioma stem cells of anaplastic oligodendroglioma (AO). This seems to be a new

ABBREVIATIONS AO = anaplastic oligodendroglioma; DAPI = diamidino-2-phenylindole; $\mathrm{EC}$ = endothelial cell; FISH = fluorescence in situ hybridization; KPS = Karnofsky Performance Scale; OS = overall survival; $P B S=$ phosphate-buffered saline; $P F S=$ progression-free survival; $R O C=$ receiver operating characteristic. 
perspective toward the mechanism of angiogenesis and chemosensitivity of oligodendroglial tumors. This study was designed to explore the origin of ECs in $1 \mathrm{p} / 19 \mathrm{q}-\mathrm{Co}-$ deleted $\mathrm{AO}$ and evaluate the prognostic significance of $1 \mathrm{p} / 19 \mathrm{q}$ co-deletion in ECs.

\section{Methods}

\section{Ethics Statement}

Records from a series of 30 patients with a histological diagnosis of primary AO and high rates of $1 \mathrm{p} / 19 \mathrm{q}$ co-deletion in the tumor cells between January 2009 and January 2010 were retrospectively retrieved from the pathology files of our department. This study was performed according to the standards of the institutional ethics committee and the Helsinki Declaration of 1975, as revised in 1983, and approved by the institutional review board of Capital Medical University.

\section{Pathological Examination}

Fresh paraffin-embedded tumor tissues were sectioned into 4- $\mu \mathrm{m}$-thick slides and stained with hematoxylin and eosin. All specimens were independently reviewed by 3 experienced neuropathologists (Jumei Wang, Guang Li, and Lin Luo), who were blinded to the clinical outcomes of the patients, according to the 2007 WHO classification for central nervous system tumors. ${ }^{10}$ In the case of a discrepancy, the 3 observers simultaneously reviewed the slides in order to achieve a consensus, and the corresponding immunohistochemical staining would be performed if necessary.

\section{Follow-Up}

Patients who underwent needle biopsies prior to resection and/or prior adjuvant therapy (radiotherapy or chemotherapy) were excluded from the analysis. This was done to create a more uniform patient population that could be propitious to the study. Progression-free survival (PFS) was designated as the time from the first operation to the time of tumor recurrence or evidence of progression on MRI. Overall survival (OS) was defined as the period between the first operation and death. The patients were followed up for 29.0 to 61.0 months after surgery, and the median follow-up was 42.0 months.

\section{Treatment}

All patients enrolled in the present study were treated according to the latest National Comprehensive Cancer Network guideline. Once pathologically diagnosed with $\mathrm{AO}$, patients received systematic chemotherapy and radiotherapy after the operation. Postoperative radiotherapy was delivered to patients within 1 month after the operation. The total dose was $60 \mathrm{~Gy}$, which was divided into 30 daily fractions of $2 \mathrm{~Gy}$ each. Meanwhile, postoperative chemotherapy was given: the common course of chemotherapy was 4 to 6 cycles, which depended on the tolerance of toxic effects. The adjuvant chemotherapy drug was mainly nimustine.

\section{Immunofluorescence}

The 4- $\mu \mathrm{m}$-thick slides were deparaffinized and de- hydrated. Sodium citrate buffer $(10 \mathrm{mM}$ sodium citrate, $0.05 \%$ Tween $20, \mathrm{pH} 6.0$ ) was added to a microwaveable vessel, and the slides were conveniently put into the microwaveable vessel. Then, we put the vessel into a microwave that was set to full power. After the solution-containing vessel was boiled for 20 minutes in the microwave, the vessel was taken out and cooled to room temperature. The slides were washed 3 times, for 3 minutes each time, in phosphate-buffered saline (PBS) with gentle agitation and then blocked in $1 \%$ bovine serum albumin for 2 hours at room temperature. After draining for a few seconds and wiping with tissue paper, the slides were incubated with the primary antibody-mouse anti-CD34 (dilution 1:100; ZM-0046, Beijing Zhongshan Golden Bridge Biotechnology Co., Ltd)-at $4^{\circ} \mathrm{C}$ overnight. Then, the slides were washed 5 times, for 3 minutes each time, in PBS with gentle agitation and incubated with the secondary antibodyrhodamine-conjugated AffiniPure goat anti-mouse IgG (1:100; ZF-0313, Beijing Zhongshan Golden Bridge Biotechnology Co., Ltd) - at $37^{\circ} \mathrm{C}$ for 2 hours. Next, the slides were rinsed 3 times, for 3 minutes each, in PBS with gentle agitation and incubated with diamidino-2-phenylindole (DAPI) at $37^{\circ} \mathrm{C}$ for 2 minutes for fluorescent detection. The last 3 steps should be done in the dark. Fluorescence was observed using an Olympus BX51TRF microscope (Olympus) (Fig. 1A).

\section{Fluorescence in Situ Hybridization}

The 1p/19q fluorescent probe kit (Vysis) was used for the fluorescence in situ hybridization (FISH) test. Briefly, the 4- $\mu \mathrm{m}$-thick paraffin slides were deparaffinized, dehydrated, and incubated in $1 \mathrm{~mol} / \mathrm{L} \mathrm{NaSCN}$ for 35 minutes at $80^{\circ} \mathrm{C}$. The slides were then immersed in a pepsin solution $(0.65 \%$ in protease buffer with $0.01 \mathrm{~mol} / \mathrm{L} \mathrm{HCl})$ for 10 minutes at $37^{\circ} \mathrm{C}$, and the tissues were fixed in $10 \%$ neutral buffered formalin. Then, the specimens were dehydrated in ethanol $(70 \%, 85 \%$, and $100 \%$ for 2 minutes in each bath), and air dried; $20 \mu \mathrm{l}$ of each probe was then added separately, and the slides were sealed with rubber cement. After co-denaturation for 10 minutes at $75^{\circ} \mathrm{C}$, the slides were then put in a humidified atmosphere with Hybrite (ThermoBriteTM; Vysis) for 16 hours at $37^{\circ} \mathrm{C}$. The slides were first immersed in $2 \times$ salt-sodium citrate/ $0.3 \%$ NP-40 for 2 minutes at room temperature and then in $2 \times$ saltsodium citrate $/ 0.3 \% \mathrm{NP}-40$ for 2 minutes at $73^{\circ} \mathrm{C}$. After drying, the nuclei were counterstained with 4,6-DAPI and an anti-fade compound (p-Phenylenediamine). The FISH signals for each locus-specific FISH probe were assessed under an Olympus BX51TRF microscope (Olympus) equipped with a triple-pass filter (DAPI/green/orange; Vysis) (Fig. 1B).

The assessment and interpretation of the FISH results were made according to the guidelines defined by the International Society of Paediatric Oncology's (SIOP) Europe Neuroblastoma Pathology and Biology and Bone Marrow Group. ${ }^{1}$ For each probe, more than 100 nonoverlapping nuclei were enumerated per hybridization. The tumor cells with more than $30 \%$ of the nuclei showing DNA loss were defined as tumor cells with chromosomal loss. According to the results of the receiver operating characteristic (ROC) curve analysis (Fig. 2), we designed the 

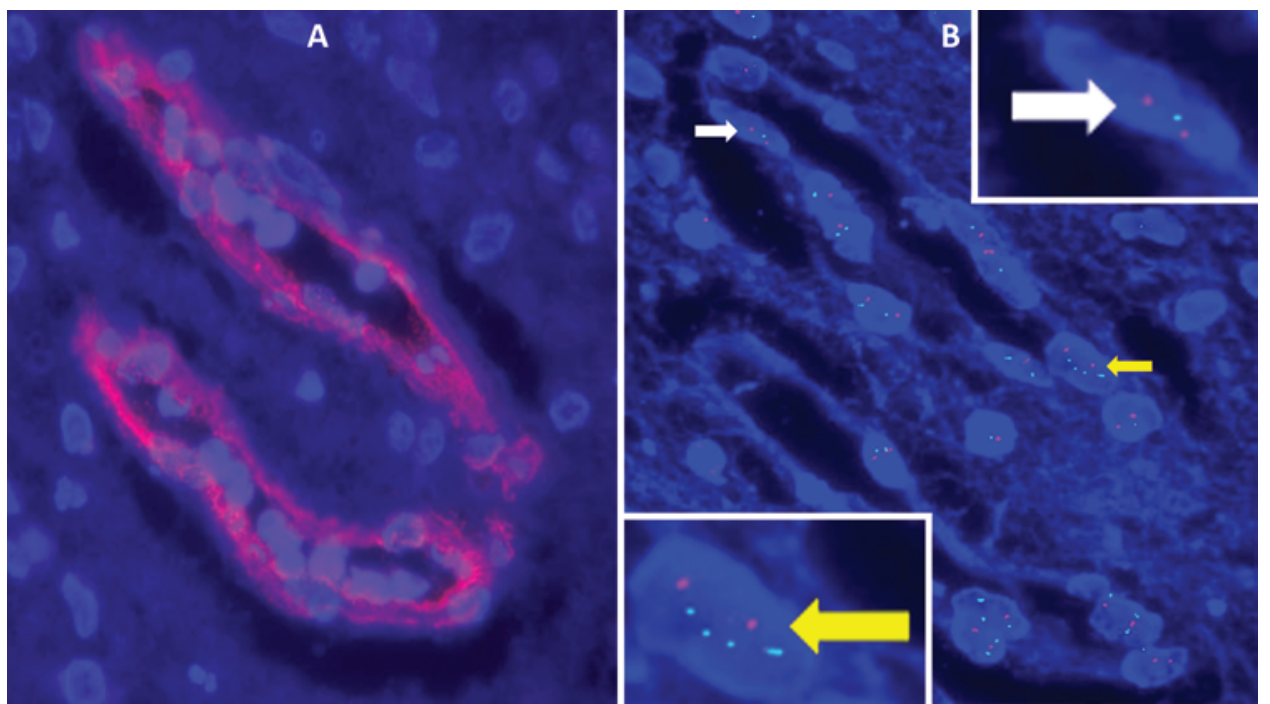

FIG. 1. Immunofluorescence detections. A: Identification of ECs. B: FISH detection results. ECs with 1p36 deletion (white arrow) and ECs with 1q25 polysomy (yellow arrow). Figure is available in color online only.

vascular ECs with more than or equal to $15 \%$ of nuclei showing DNA loss as ECs with $1 \mathrm{p} / 19 \mathrm{q}$ deletion, while those with less than $15 \%$ were defined as $1 p / 19 q$ intact.

\section{Statistical Analysis}

The statistical analysis was performed using SPSS 13.0 for Windows. Survival function concerning the $1 p / 19 q$ deletion status of the vascular ECs was assessed using the Kaplan-Meier estimate and log-rank test. The pairedsample t-test was used to compare the $1 \mathrm{p} / 19 \mathrm{q}$ deletion rate between $1 \mathrm{p} 36$ and $1 \mathrm{q} 25$ or $19 \mathrm{q} 13$ and $19 \mathrm{p} 13$. The $\mathrm{p}$ value was obtained using 2 -sided tests with a statistical significance of $\mathrm{p}<0.05$. The appropriate $1 \mathrm{p} / 19 \mathrm{q}$ deletion cutoff values for vascular ECs for distinguishing long-term survivors from short-term survivors were designed according to the area under the ROC curve. Generally, an area under the ROC curve $\geq 0.70$ was considered a clinically useful predictive model.

\section{Results}

\section{Basic Characteristics}

The basic clinical characteristics of the patients en-

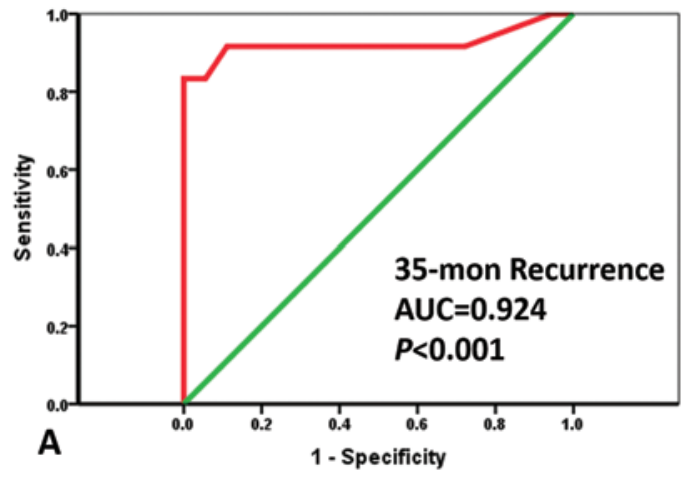

rolled in this study are summarized in Table 1. A total of 30 patients with a diagnosis of primary AO who were surgically treated at our center were included in the present study. There were 16 male and 14 female patients with a median age of 46.5 years (range 23-61 years). The median Karnofsky Performance Scale (KPS) score of this cohort was 90 (range 70-100).

Gross-total resection was achieved in $21(70.0 \%)$ patients, subtotal resection in $8(26.7 \%)$ patients, and partial resection in $1(3.3 \%)$ patient.

There were $15(50.0 \%)$ AOs located in the frontal lobe, $12(40.0 \%)$ in the temporal lobe, $2(6.7 \%)$ in the parietal lobe, and $1(3.3 \%)$ in the insular cortex.

\section{1p/19q Co-Deletion in the Vascular ECs of AO}

For each case, the tissue was cut into 4- $\mu$ m-thick serial sections. We chose 1 slide for immunofluorescence detection in order to locate the ECs (Fig. 1A), while another slide close to it was chosen for FISH detection with the aim of evaluating $1 \mathrm{p} / 19 \mathrm{q}$ status (Fig. 1B).

The ECs in AO had a higher 1p36 (the detected signal) deletion rate than $1 \mathrm{q} 25$ (the reference signal) $(\mathrm{p}<0.01)$

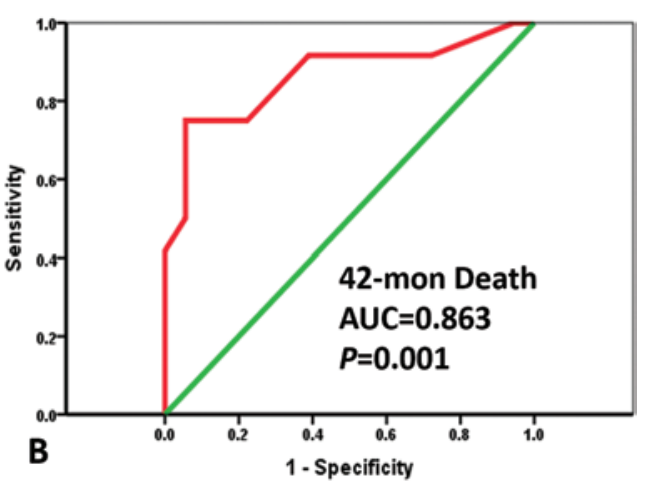

FIG. 2. ROC curves of the accuracy of using the cutoff value of $15 \%$ as the criterion for $1 p / 19 q$ deletion in ECs. The plot was constructed by computing the sensitivity versus $1-$ specificity for the possible cutoff point of the $1 p / 19 q$ deletion rate in ECs. AUC = area under the curve. Figure is available in color online only. 
TABLE 1. Summary of the clinical characteristics of AO patients

\begin{tabular}{|c|c|c|c|c|c|c|c|}
\hline Case No. & Sex & Age (yrs) & Location & Degree of Resection & KPS Score & $\begin{array}{l}\text { Recurrence } \\
\text { PFS (mos) }\end{array}$ & $\begin{array}{c}\text { Death } \\
\text { OS (mos) }\end{array}$ \\
\hline 1 & M & 34 & Frontal & GTR & 90 & 36.0 & 44.0 \\
\hline 2 & M & 23 & Frontal & STR & 100 & 46.5 & 53.0 \\
\hline 3 & $\mathrm{~F}$ & 57 & Temporal & GTR & 90 & 33.5 & 47.0 \\
\hline 4 & $M$ & 28 & Temporal & GTR & 80 & 44.5 & 54.0 \\
\hline 5 & $\mathrm{~F}$ & 43 & Temporal & GTR & 90 & 38.0 & 42.0 \\
\hline 6 & $\mathrm{~F}$ & 51 & Frontal & STR & 100 & 35.0 & 41.0 \\
\hline 7 & $\mathrm{~F}$ & 48 & Parietal & GTR & 70 & 41.5 & 49.0 \\
\hline 8 & M & 58 & Frontal & STR & 90 & 35.0 & 39.0 \\
\hline 9 & M & 38 & Frontal & GTR & 80 & 29.0 & 32.5 \\
\hline 10 & $\mathrm{~F}$ & 45 & Temporal & GTR & 80 & 44.0 & 52.0 \\
\hline 11 & $M$ & 56 & Frontal & GTR & 100 & 35.0 & 36.0 \\
\hline 12 & $M$ & 45 & Frontal & STR & 90 & 47.0 & 56.0 \\
\hline 13 & $\mathrm{~F}$ & 48 & Temporal & GTR & 100 & 32.0 & 38.0 \\
\hline 14 & $\mathrm{~F}$ & 52 & Parietal & STR & 80 & 28.0 & 42.0 \\
\hline 15 & M & 36 & Frontal & GTR & 100 & 49.5 & 57.5 \\
\hline 16 & $M$ & 54 & Temporal & STR & 70 & 29.0 & 34.0 \\
\hline 17 & M & 51 & Temporal & GTR & 90 & 30.0 & 35.0 \\
\hline 18 & $\mathrm{~F}$ & 41 & Frontal & GTR & 90 & 32.0 & 42.0 \\
\hline 19 & $\mathrm{~F}$ & 55 & Frontal & GTR & 90 & 35.0 & 43.0 \\
\hline 20 & M & 43 & Insular & PR & 100 & 26.0 & 29.0 \\
\hline 21 & $\mathrm{~F}$ & 55 & Frontal & GTR & 70 & 35.0 & 39.5 \\
\hline 22 & $M$ & 61 & Temporal & GTR & 80 & 32.0 & 38.0 \\
\hline 23 & M & 43 & Frontal & GTR & 90 & 30.5 & 40.0 \\
\hline 24 & $\mathrm{M}$ & 48 & Temporal & GTR & 80 & 31.0 & 41.0 \\
\hline 25 & $\mathrm{~F}$ & 32 & Temporal & GTR & 90 & 42.0 & 49.5 \\
\hline 26 & $\mathrm{~F}$ & 58 & Frontal & STR & 90 & 32.0 & 38.0 \\
\hline 27 & $M$ & 43 & Frontal & GTR & 100 & 41.0 & 43.0 \\
\hline 28 & $F$ & 52 & Temporal & STR & 70 & 27.5 & 40.0 \\
\hline 29 & $\mathrm{~F}$ & 37 & Frontal & GTR & 80 & 48.5 & 61.0 \\
\hline 30 & M & 41 & Temporal & GTR & 90 & 34.0 & 42.0 \\
\hline
\end{tabular}

GTR = gross-total resection; PR = partial resection; STR = subtotal resection.

and a higher $19 \mathrm{q} 13$ (the detected signal) deletion rate than $19 \mathrm{p} 13$ (the reference signal) $(\mathrm{p}<0.01)$ (Table 2).

We performed ROC analysis to determine the accuracy of using $15 \%$ as the cutoff for $1 p / 19 q$ deletion in ECs (Fig. 2). Meanwhile, the median PFS and OS of the whole cohort enrolled in this study were 35.0 months and 42.0 months, respectively. Therefore, recurrence at 35 months and death at 42 months were selected to serve as observation points. The ROC curves for $1 \mathrm{p} / 19 \mathrm{q}$ deletion were plotted. We observed clear separations between the groups of patients with a $1 p / 19 q$ deletion rate $\geq 15 \%$ in ECs and those with a $1 \mathrm{p} / 19 \mathrm{q}$ deletion rate $<15 \%$ in ECs with respect to both PFS and OS, with areas under the curve of 0.924 (95\% CI $0.791-1.000)$ for PFS and 0.863 (95\% CI $0.714-1.000$ ) for OS. Corresponding to this analysis, the cutoff $1 p / 19 q$ deletion rate with the highest accuracy for both PFS and OS was determined as follows: $1 \mathrm{p} / 19 \mathrm{q}$ deletion rate of $15 \%$ with $83.3 \%$ sensitivity and $100.0 \%$ specificity for PFS, and $1 \mathrm{p} / 19 \mathrm{q}$ deletion rate of $15 \%$ with
$75.0 \%$ sensitivity and $94.4 \%$ specificity for OS (Fig. 2). According to our specific co-deletion criteria, the patients could be divided into long-term survivors and short-term survivors, and 10 of $30(33.3 \%)$ patients exhibited $1 \mathrm{p} / 19 \mathrm{q}$ co-deletion in ECs.

\section{Prognostic Implication of $1 \mathrm{p} / 19 q$ Co-Deletion in Vascular ECs}

1p/19q co-deletion in ECs was associated with longer PFS and OS. Patients who harbored $1 \mathrm{p} / 19 \mathrm{q}-$ co-deleted ECs demonstrated recurrence with a median PFS of 44.0 months, which was significantly longer than those with $1 p / 19 q-i n t a c t$ ECs $(p<0.001)$. Furthermore, patients who harbored 1p/19q-co-deleted ECs died at a median OS of 52.0 months, which was significantly longer than those with 1p/19q-intact ECs ( $\mathrm{p}<0.001)$. Our results were validated in an independent cohort that contained $30 \mathrm{AO}$ samples from the Beijing Neurosurgical Institute. According to the $1 \mathrm{p} / 19 \mathrm{q}$ deletion rate of the ECs, the samples of the 
TABLE 2. The $1 p / 19 q$ deletion rate of vascular ECs in $A O$

\begin{tabular}{|c|c|c|c|c|}
\hline \multirow[b]{2}{*}{$\begin{array}{c}\text { Case } \\
\text { No. }\end{array}$} & \multicolumn{2}{|c|}{ Subgroup 1} & \multicolumn{2}{|c|}{ Subgroup 2} \\
\hline & $\begin{array}{c}\text { 1q25 } \\
\text { Deletion } \\
\text { Rate }(\%)\end{array}$ & $\begin{array}{c}\text { 1p36 } \\
\text { Deletion } \\
\text { Rate }(\%)\end{array}$ & $\begin{array}{c}\text { 19p13 } \\
\text { Deletion } \\
\text { Rate }(\%)\end{array}$ & $\begin{array}{c}19 q 13 \\
\text { Deletion } \\
\text { Rate }(\%)\end{array}$ \\
\hline 1 & 4 & 17 & 4 & 16 \\
\hline 2 & 4 & 19 & 5 & 17 \\
\hline 3 & 3 & 15 & 1 & 12 \\
\hline 4 & 6 & 26 & 5 & 18 \\
\hline 5 & 2 & 17 & 4 & 16 \\
\hline 6 & 4 & 11 & 2 & 23 \\
\hline 7 & 7 & 16 & 1 & 15 \\
\hline 8 & 8 & 12 & 3 & 11 \\
\hline 9 & 5 & 12 & 2 & 15 \\
\hline 10 & 4 & 17 & 2 & 25 \\
\hline 11 & 4 & 10 & 7 & 10 \\
\hline 12 & 0 & 15 & 3 & 18 \\
\hline 13 & 5 & 12 & 12 & 5 \\
\hline 14 & 6 & 7 & 4 & 11 \\
\hline 15 & 2 & 22 & 4 & 23 \\
\hline 16 & 2 & 12 & 9 & 7 \\
\hline 17 & 9 & 13 & 6 & 19 \\
\hline 18 & 2 & 16 & 11 & 8 \\
\hline 19 & 2 & 12 & 1 & 14 \\
\hline 20 & 1 & 10 & 0 & 8 \\
\hline 21 & 5 & 10 & 4 & 7 \\
\hline 22 & 3 & 10 & 5 & 15 \\
\hline 23 & 7 & 7 & 4 & 14 \\
\hline 24 & 4 & 15 & 7 & 13 \\
\hline 25 & 15 & 15 & 9 & 18 \\
\hline 26 & 0 & 19 & 3 & 14 \\
\hline 27 & 5 & 7 & 6 & 25 \\
\hline 28 & 1 & 12 & 6 & 15 \\
\hline 29 & 7 & 21 & 4 & 21 \\
\hline 30 & 0 & 26 & 3 & 12 \\
\hline$p$ value & \multicolumn{2}{|c|}{$<0.01$} & \multicolumn{2}{|c|}{$<0.01$} \\
\hline
\end{tabular}

independent validation cohort were divided into 2 groups: $1 \mathrm{p} / 19 \mathrm{q}$ deletion rate $\geq 15 \%$ (i.e., $1 \mathrm{p} / 19 \mathrm{q}$ co-deletion) and $1 \mathrm{p} / 19 \mathrm{q}$ deletion rate $<15 \%$ (i.e., $1 \mathrm{p} / 19 \mathrm{q}$ intact). The results of the survival analysis of the 2 groups demonstrated that there was a statistically significant difference in prognosis ( $p<0.001$ for PFS and $p=0.003$ for OS) (Fig. 3).

In a multivariate Cox regression model that included age, KPS, degree of tumor resection, and $1 \mathrm{p} / 19 \mathrm{q}$ status, $1 \mathrm{p} / 19 \mathrm{q}$ codeletion in ECs was confirmed as an independent prognostic factor and was associated with both longer PFS and OS. The hazard ratio of $1 \mathrm{p} / 19 \mathrm{q}$ co-deletion in ECs was $0.056(95 \%$ CI $0.012-0.261 ; \mathrm{p}<0.001)$ for PFS and 0.061 (95\% CI $0.013-0.280 ; \mathrm{p}<0.001)$ for OS.

\section{Discussion}

It has been well established that $1 \mathrm{p} / 19 \mathrm{q}$ co-deletion is a genetic marker for oligodendroglial tumors and confers favorable chemosensitivity and prognosis. In 1998, Cairncross et al. reported that the loss of $1 \mathrm{p}$ and/or $1 \mathrm{p} / 19 \mathrm{q}$ co-deletion predicted a better response to procarbazine-lomustine-vincristine chemotherapy and a longer survival time in patients with $\mathrm{AO}{ }^{3}$ These findings have been reproduced in many subsequent studies, including prospective and randomized Phase III clinical trials. ${ }^{2,18}$ However, the prognostic significance of $1 p / 19 q$ co-deletion was elaborated on the basis of a theory that the existence of $1 p / 19 q$ co-deletion was found only in tumor cells. In other words, $1 \mathrm{p} / 19 \mathrm{q}$ codeletion was a tumor cell-specific biomarker. However, in the present study, we found some ECs in AO possessed this chromosomal abnormality as well. Furthermore, 1p/19q co-deletion in ECs also conferred a longer survival time. This might be a new perspective toward the vascular development and maintenance of malignant gliomas.

\section{Vascular ECs Might Be Derived From Glioma Stem Cells}

The formation of blood vessels in glioma is extremely complicated. It has been reported that glioma stem cells could differentiate into vascular ECs, which assemble to form a vascular plexus that could support blood cell circulation and mature into a vascular network. ${ }^{6}$ Using the patients' surgical specimens, Wang et al. ${ }^{17}$ and Ricci-Vitiani et al. ${ }^{13}$ found a subset of ECs lining the tumor vessels that carried the same genetic abnormalities (e.g., amplification of epidermal growth factor receptor or TP53 mutation), which were exclusive markers of adjacent malignant tumor cells. These 2 research groups both showed that a subpopulation of glioma stem cells could give rise to endothelial cells in vitro. These cells, when intracranially transplanted into immunocompromised mice, could give rise to tumors. Further research confirmed that in these tumors there was a proportion of ECs that contributed to blood vessels and originated from the transplanted human glioma cells rather than from mice. Soda et al. ${ }^{15}$ observed a subpopulation of ECs within these tumors that could express both EC-specific and tumor-specific antigens.

In the present study, we revealed that $1 \mathrm{p} / 19 \mathrm{q}$ co-deletion, which was initially regarded as a tumor cell-specific chromosomal abnormality, could also be found in ECs. However, not all (100\%) ECs contained the 1p/19q deletion signature. This was consistent with Streubel and colleagues' report, which found that $15 \%$ to $85 \%$ (median $37 \%$ ) of the microvascular ECs in B-cell lymphomas harbored lymphoma-specific chromosomal translocations. ${ }^{16}$ All of these findings implied that the ECs and tumor cells in gliomas share a common pathway in tumorigenesis. Perhaps in the process of glioma stem cells differentiating into ECs and tumor cells, this genetic signature was delivered at the same time. As for the phenomenon that some ECs did not appear to exhibit $1 \mathrm{p} / 19 \mathrm{q}$ deletion, the sectioning of tumor samples from different directions, to some extent, could influence the experimental results, especially when judging the $1 p / 19 q$ status of the small vessels, which could partly contribute to it.

\section{1p/19q Co-Deletion in ECs Conferred a Favorable Prognosis}

The $1 \mathrm{p} / 19 \mathrm{q}$ deletion rate in ECs was relatively lower 

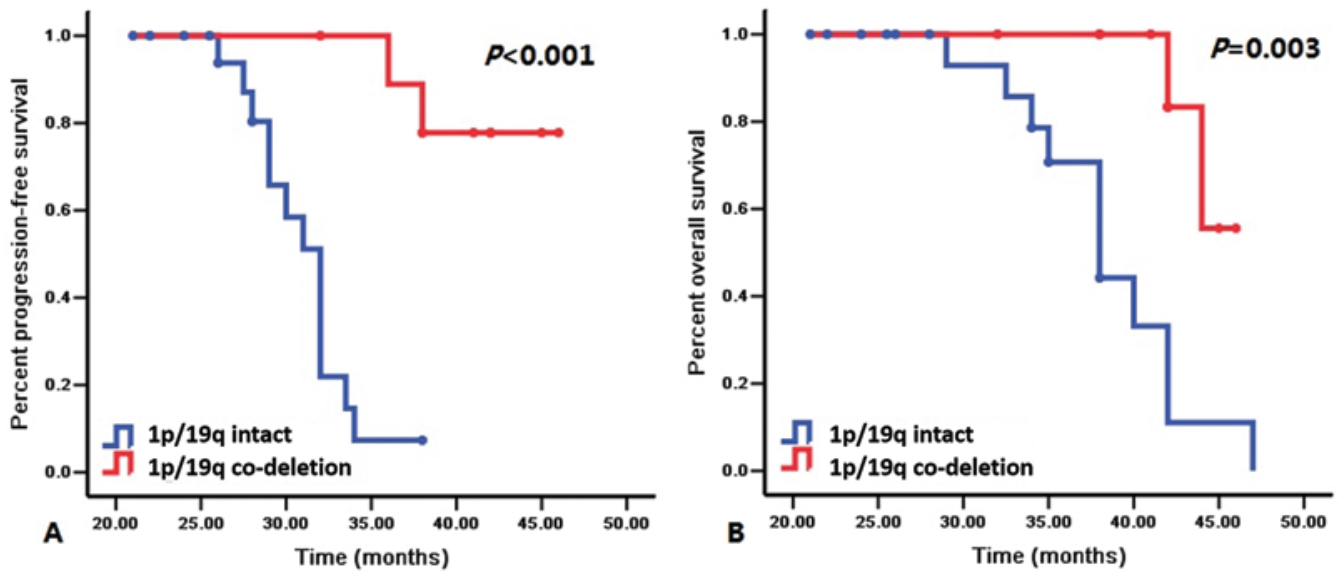

FIG. 3. In an independent validation cohort, the survival analysis showed that the 2 groups divided by the $1 p / 19 q$ deletion rate was significantly different ( $p<0.001$ for PFS and $p=0.003$ for OS). Figure is available in color online only.

than that in tumor cells. According to the results of the ROC analysis, we defined the cutoff of $15 \%$ as the criterion for $1 \mathrm{p} / 19 \mathrm{q}$ deletion in ECs. Therefore, in this study, 10 of $30(33.3 \%)$ 1p/19q-co-deleted AOs exhibited 1p/19q co-deletion in ECs.

Our study, for the first time, interpreted that the survival time of patients with $1 \mathrm{p} / 19 \mathrm{q}-$-co-deleted ECs is significantly longer than those with $1 \mathrm{p} / 19 \mathrm{q}$-intact ECs, which was further validated in an independent cohort. Although $1 \mathrm{p} / 19 \mathrm{q}$ co-deletion is a well-known genetic marker of favorable clinical outcome, all of those former results were only elaborated in tumor cells. The favorable clinical outcomes of patients with $1 \mathrm{p} / 19 \mathrm{q}-\mathrm{co}-$ deleted tumor cells resulted from relatively better chemosensitivity and radiosensitivity. ${ }^{2,18}$ As for the patients with $1 \mathrm{p} / 19 \mathrm{q}$ co-deleted $\mathrm{ECs}$, the reasons for the prognosis advantage require further inquiry.

\section{Potential Mechanism of Resistance to Antiangiogenesis Therapy}

According to the FISH detection results, 20 (66.7\%) of 30 patients had 1p/19q-intact ECs, which conferred a dismal prognosis. Perhaps the dismal prognosis of this subgroup was attributable to the limited sensitivities of $1 \mathrm{p} / 19 \mathrm{q}$-intact ECs to therapies, including chemotherapy and antiangiogenesis treatment. With regard to the compromised effectiveness of antiangiogenesis therapy, Cheng et $a{ }^{4}{ }^{4}$ found that glioma stem cells could also generate some vascular pericytes that surrounded blood vessels within the glioblastoma multiforme. Furthermore, they observed that the majority of vascular pericytes carried the same genetic alterations, which were found in the glioblastoma multiforme. These findings had important implications for glioblastoma therapy: increased pericyte coverage was thought to have conferred resistance to bevacizumab.

In our previous studies, we have found that $1 \mathrm{q}$ and/or $19 p$ polysomy in the tumor cells of glioma showed an unfavorable outcome. ${ }^{9,12}$ According to the FISH detection results, we noted that the tumor cell-specific chromosomal aberrations (1q and 19p polysomy) also occurred in the ECs (Fig. 1). In 2004, Streubel et al. ${ }^{16}$ reported that numerical chromosomal aberrations were shared by lymphoma cells and ECs. As we all know, polysomy was always treated as a consequence of increased genetic instability and cellcycle dysregulation, which might lead to increased copy numbers of chromosomes and then correlate with tumor progression. Therefore, the existence of ECs harboring polysomy might be another potential reason for resistance to antiangiogenesis drugs. But, this idea remains to be elucidated in future research.

\section{Study Limitations}

Some limitations existed in this study. There were only 30 patients in this series, which is not so large. Besides, the potential molecular mechanisms of $1 \mathrm{p} / 19 \mathrm{q}$ co-deletion in ECs associated with favorable prognosis have not been disclosed. In the future, we will expand the sample and continue carrying out this research.

\section{Conclusions}

1p/19q co-deletion and polysomy are shared by tumor cells and ECs in AO. Our findings suggest that vascular $\mathrm{ECs}$ in $\mathrm{AO}$ are, in part, tumor related, which points to a novel aspect of tumor angiogenesis.

\section{Acknowledgments}

We acknowledge financial support by Beijing Natural Science Foundation (7122061), National Natural Science Foundation of China (81401381), and the Capital Health Research and Development Special Fund (2014-2-2042). We thank Dr. Lin Luo for providing pathological diagnoses, Department of Pathology, Beijing Neurosurgical Institute; and Dr. Guang Li, Department of Pathology, Beijing Tiantan Hospital, Capital Medical University.

\section{References}

1. Ambros PF, Ambros IM: Pathology and biology guidelines for resectable and unresectable neuroblastic tumors and bone marrow examination guidelines. Med Pediatr Oncol 37:492-504, 2001

2. Cairncross G, Berkey B, Shaw E, Jenkins R, Scheithauer $\mathrm{B}$, Brachman D, et al: Phase III trial of chemotherapy plus 
radiotherapy compared with radiotherapy alone for pure and mixed anaplastic oligodendroglioma: Intergroup Radiation Therapy Oncology Group Trial 9402. J Clin Oncol 24:27072714, 2006

3. Cairncross JG, Ueki K, Zlatescu MC, Lisle DK, Finkelstein $\mathrm{DM}$, Hammond RR, et al: Specific genetic predictors of chemotherapeutic response and survival in patients with anaplastic oligodendrogliomas. J Natl Cancer Inst 90:1473-1479, 1998

4. Cheng L, Huang Z, Zhou W, Wu Q, Donnola S, Liu JK, et al: Glioblastoma stem cells generate vascular pericytes to support vessel function and tumor growth. Cell 153:139-152, 2013

5. Chinot OL, Wick W, Mason W, Henriksson R, Saran F, Nishikawa R, et al: Bevacizumab plus radiotherapy-temozolomide for newly diagnosed glioblastoma. N Engl J Med 370:709-722, 2014

6. Das S, Marsden PA: Angiogenesis in glioblastoma. N Engl J Med 369:1561-1563, 2013

7. Gilbert MR, Dignam JJ, Armstrong TS, Wefel JS, Blumenthal DT, Vogelbaum MA, et al: A randomized trial of bevacizumab for newly diagnosed glioblastoma. N Engl J Med 370:699-708, 2014

8. Hirst TC, Vesterinen HM, Sena ES, Egan KJ, Macleod MR, Whittle IR: Systematic review and meta-analysis of temozolomide in animal models of glioma: was clinical efficacy predicted? Br J Cancer 108:64-71, 2013

9. Jiang H, Ren X, Wang J, Zhang Z, Jia W, Lin S: Short-term survivors in glioblastomas with oligodendroglioma component: a clinical study of 186 Chinese patients from a single institution. J Neurooncol 116:395-404, 2014

10. Louis DN, Ohgaki H, Wiestler OD, Cavenee WK, Burger PC, Jouvet A, et al: The 2007 WHO classification of tumours of the central nervous system. Acta Neuropathol 114:97-109, 2007

11. Pàez-Ribes M, Allen E, Hudock J, Takeda T, Okuyama H, Viñals F, et al: Antiangiogenic therapy elicits malignant progression of tumors to increased local invasion and distant metastasis. Cancer Cell 15:220-231, 2009

12. Ren X, Jiang H, Cui X, Cui Y, Ma J, Jiang Z, et al: Co-polysomy of chromosome 1q and 19p predicts worse prognosis in $1 \mathrm{p} / 19 \mathrm{q}$ codeleted oligodendroglial tumors: FISH analysis of 148 consecutive cases. Neuro Oncol 15:1244-1250, 2013

13. Ricci-Vitiani L, Pallini R, Biffoni M, Todaro M, Invernici G,
Cenci T, et al: Tumour vascularization via endothelial differentiation of glioblastoma stem-like cells. Nature 468:824828,2010

14. Schwartzbaum JA, Fisher JL, Aldape KD, Wrensch M: Epidemiology and molecular pathology of glioma. Nat Clin Pract Neurol 2:494-503, quiz 1, 516, 2006

15. Soda Y, Marumoto T, Friedmann-Morvinski D, Soda M, Liu F, Michiue H, et al: Transdifferentiation of glioblastoma cells into vascular endothelial cells. Proc Natl Acad Sci U S A 108:4274-4280, 2011

16. Streubel B, Chott A, Huber D, Exner M, Jäger U, Wagner O, et al: Lymphoma-specific genetic aberrations in microvascular endothelial cells in B-cell lymphomas. N Engl J Med 351:250-259, 2004

17. Wang R, Chadalavada K, Wilshire J, Kowalik U, Hovinga $\mathrm{KE}$, Geber A, et al: Glioblastoma stem-like cells give rise to tumour endothelium. Nature 468:829-833, 2010

18. Wick W, Hartmann C, Engel C, Stoffels M, Felsberg J, Stockhammer F, et al: NOA-04 randomized phase III trial of sequential radiochemotherapy of anaplastic glioma with procarbazine, lomustine, and vincristine or temozolomide. J Clin Oncol 27:5874-5880, 2009

\section{Disclosures}

The authors report no conflict of interest concerning the materials or methods used in this study or the findings specified in this paper.

\section{Author Contributions}

Conception and design: Jiang. Acquisition of data: Zhang, Zeng. Analysis and interpretation of data: Zhang. Drafting the article: Jiang. Critically revising the article: Ren. Reviewed submitted version of manuscript: Ren. Approved the final version of the manuscript on behalf of all authors: Lin. Statistical analysis: Zeng. Administrative/technical/material support: Wang. Study supervision: Lin.

\section{Correspondence}

Song Lin, Neurosurgery, Beijing Tiantan Hospital, Capital Medical University, No. 6 Tiantan Xili, Chongwen District, Beijing 100050, China. email: linsong2005@126.com. 